\title{
SIAT - Sistema de Avaliação de Terras ${ }^{1}$
}

\author{
Gilberto J. Garcia² \& Carlos R. Espindola ${ }^{3}$
}

1 Projeto desenvolvido com recursos da FAPESP - Fundação de Amparo à Pesquisa do Estado de São Paulo

2 Departamento de Planejamento Territorial, IGCE - UNESP, Rio Claro, SP, Brasil. E-mail: gilberto@rc.unesp.br (Foto)

${ }^{3}$ Departamento de Solos, Faculdade de Engenharia Agrícola, UNICAMP, Campinas, SP, Brasil

\begin{abstract}
Resumo: O presente trabalho teve como objetivo adaptar o sistema MicroLEIS - Land Evaluation Information System de origem espanhola, às condições do sudeste brasileiro; para tanto, foram consideradas 12 variáveis, discriminadas a seguir: A - Fator Relevo: 1) Declividade; B - Fator Solo: 2) Profundidade Útil; 3) Tipo de Textura; 4) Pedregosidade e/ou Afloramento; 5) Tipo de Drenagem; 6) Salinidade Total; C - Fator Risco à Erosão: 7) Erodibilidade; 8) Declividade; 9) Densidade da Vegetação; 10) Erosividade da Chuva; D - Fator Deficiência Bioclimática: 11) Risco de Geadas e 12) Água Disponível. Para estas variáveis, foram modificados os parâmetros de controle, de acordo com as condições dos solos paulistas, sendo que Salinidade Total foi trocada por Acidez $(\mathrm{pH})$; em seguida, o SIAT foi interfaceado com um SIG - Sistema de Informação Geográfica - no caso o IDRISI, para facilitar a entrada, o processamento e a espacialização dos resultados. A estrutura do programa também foi modificada, de maneira que a linguagem de programação passou de Basic para C, além de receber um banco de dados (Clipper) e rotinas para tornar o programa mais amigável. O programa modificado foi testado na região de Leme, SP, onde mostrou que, embora proporcione resultados satisfatórios em escalas grandes, é mais indicado em avaliações regionais para escalas entre 1:100.000 e 1:50.000.
\end{abstract}

Palavras-chave: aptidão agrícola, geoprocessamento, solo, clima

\section{SIAT - Land Evaluation System}

\begin{abstract}
The purpose of this work was to adapt the MicroLEIS - Land Evaluation Information System developed in Spain to the Brazilian Southeast conditions. Twelve (12) variables were considered, as follows: A - Relief Factor: 1) Slope; B - Soil Factor: 2) Effective depth, 3) Texture, 4) Stoniness, 5) Drainage, 6) Salinity; C - Erosion Factor: 7) Erodibility, 8) Slope, 9) Vegetation density, 10) Rainfall erosivity; D - Bioclimatic Deficiency Factor: 11) Frost, 12) Available water. For each of them, the control parameters were changed, according to conditions of the soils of the State of São Paulo. In this case, salinity was substituted by the $\mathrm{pH}$. As a second step, the SIAT was interfaced with the GIS - Geographic Information System - IDRISI, to facilitate the data input, processing and spatialization of the results. The program structure was changed from Basic to C, and a database (Clipper) and routines were introduced so as to make the system more friendly. The modified system was tested in the Leme - SP region, showing good results in larger scale, although it is more indicated for regional evaluations at scales between 1:100,000 and 1:50,000.
\end{abstract}

Key words: land suitability, geoprocessing, soil, climate

\section{INTRODUÇÃO}

Características diversas, como heterogeneidade, dimensão e desenvolvimento regional diferenciado, fazem com que o Brasil apresente situações amplamente antagônicas no que se refere à tecnificação da atividade agrícola. Nesse contexto verificam-se em regiões de São Paulo e Paraná, solos muito degradados, ao lado de áreas de manejo altamente tecnificado. Na região norte, extensas áreas estão sendo desmatadas, sem que tenham merecido um estudo sério sobre a melhor utilização das terras.
Em determinados casos, os técnicos intervêm após a ação antrópica já ter atingido grau impactante significativo, já que o governo é extremamente lento na tomada de decisões. De modo geral, os recursos naturais têm sido utilizados de forma tão acelerada e intensa, que as intervenções do mundo científico e da sociedade tem sido mais no sentido de reabilitar áreas degradadas do que prevenir problemas ambientais.

O planejamento do uso da terra no território brasileiro tem utilizado como, ferramentas, as seguintes classificações técnicas interpretativas: a) Capacidade de Uso das Terras: 
práticas de conservação do solo, a nível de propriedade; b) Aptidão Agrícola: regionalização de diferentes tipos de utilização agrícola; c) Zoneamento Ecológico: reordenamento e implantação de culturas e da cobertura vegetal. Dentro essas modalidades, citam-se os trabalhos desenvolvidos por Vieira et al. (1976), Secretaria da Agricultura (1976), Ramalho et al. (1978), SUPLAN (1979), Secretaria da Indústria e Comércio (1980), Lepsch (1983) e Oliveira \& Berg (1985). Em qualquer uma das três modalidades, a automatização é uma condição básica quando se trata de agilizar os trabalhos e oferecer, à sociedade, respostas rápidas e de baixo custo.

$\mathrm{Na}$ informatização dos procedimentos, fica evidente a necessidade de se trabalhar com números, de modo que todas as informações de entrada no modelo devem representar uma quantidade ou proporcionalidade, dentro de um universo preestabelecido. Os sistemas de informatização de dados das terras, conquanto ainda novidade no Brasil, têm merecido grande atenção da comunidade científica internacional, podendo-se referir, neste particular, os trabalhos de Anderson (1987), Lanen et al. (1988), Lindhult et al. (1988), Baudry (1989), Elliot \& Cole (1989), Ferrari \& Magaldi (1989), Lai (1990)e Schrey (1992).

A modelagem matemática, cuja ênfase aparece nos anos 80 , pode tratar de diferentes aspectos do meio ambiente e vem sendo gradativamente associada aos Sistemas de Informação Geográfica, pela facilidade de entrada, processamento e recuperação/espacialização dos dados. Como exemplo, podem ser citados Keulen (1990), ITC (1990), Lai (1990), Frank et al (1991), MacClean et al. (1993) e Koffler (1993). Dentre os inúmeros SIG's existentes, o IDRISI (Eastman, 1977) é amplamente utilizado, especialmente pela simplicidade, custo e facilidade operacional.

O presente trabalho teve como objetivo a adaptação do software espanhol MicroLEIS - Land Evaluation Information System, às condições do sudeste brasileiro, em especial o programa CERVATANA, que é específico para identificação e mapeamento da capacidade de uso das terras, passando a se chamar Sistema de Avaliação de Terras - SIAT.

\section{MATERIAL E MÉTODOS}

\section{Área de estudo}

Para testar as adaptações no Sistema, selecionou-se uma área cujas coordenadas são $22^{\circ} 00^{\prime}$ e $22^{\circ} 15^{\prime}$ de Latitude Sul e $47^{\circ} 15^{\prime}$ e $47^{\circ} 30^{\prime}$ de Longitude Oeste, correspondendo à Folha Leme do IBGE, na escala 1:50.000.

A geologia da região é representada por rochas relacionadas com o Grupo Tubarão do permocarbonífero, com as formações Irati e Corumbataí, do grupo Passa-Dois, com intrusivas básicas, com arenitos da formação Botucatu-Pirambóia e com o Cenozóico (IPT, 1981).

O clima, segundo o sistema Koppen, é do tipo Cwa, ou seja, subtropical, seco no inverno e chuvoso no verão, com temperatura média do mês mais quente superior a $22^{\circ} \mathrm{C}$ e a do mês mais frio, abaixo de $18^{\circ} \mathrm{C}$ (Setzer, 1966).

O relevo predominante é suavemente ondulado mostrando, no entanto, áreas significativas de ondulado e pequenas áreas fortemente onduladas; quanto à vegetação natural é escassa, resumindo-se basicamente a matas galerias, vegetação arbórea de encosta e vegetação de terras úmidas. A área é intensamente cultivada, em especial com cana-de-açúcar, citrus, culturas anuais, como algodão, milho e soja, além de algumas áreas de reflorestamento.

Quanto aos solos, mapeados por Oliveira et al. (1982) e reclassificados de acordo com EMBRAPA (1999) tem-se os seguintes: Latossolos Vermelho-Amarelos, Latossolos Vermelhos, Argissolos Vermelho-Amarelos, Nitossolos Vermelhos, Neossolos Quatzarênicos e Gleissolos.

\section{O sistema MicroLEIS}

O sistema MicroLEIS foi desenvolvido para avaliação ecológica de terras com fins ao uso agrícola, pastoril, florestal e de reservas naturais, em regiões mediterrâneas. Este programa automatiza os procedimentos da aplicação de um sistema de avaliação de terras para fins agrícolas (Rosa et al., 1992). O programa CERVATANA é parte do sistema MicroLEIS e ordena as informações sobre a aptidão das terras, utilizando a seguinte nomenclatura (classes):

$\mathrm{S}_{1}$ : Excelente capacidade de uso; terras com alta qualidade agrológica, com nenhuma ou poucas limitações que restrinjam seu uso.

$\mathrm{S}_{2}$ : Boa capacidade de uso; apresentam algumas limitações de ordem topográfica, edáfica ou climática, restringindo o número de cultivos possíveis, assim como a capacidade produtiva.

$\mathrm{S}_{3}$ : Moderada capacidade de uso; apresentam limitações importantes relacionadas com fatores topográfico, edáfico ou climático; as técnicas de manejo são mais difíceis de serem aplicadas e mantidas.

N - Marginais ou improdutivas; aptas somente para pastagens ou produção florestal; esta classe inclui também as terras totalmente improdutivas.

Com exceção da classe $\mathrm{S}_{1}$, todas as outras se dividem em subclasses, com base em um ou mais dos seguintes critérios diagnóstico: Fator Relevo; Fator Solo: Profundidade, Textura, Pedregosidade e/ou afloramentos, Drenagem, Salinidade; Fator Risco de Erosão: Erodibilidade, Declividade, Densidade da Vegetação, Erosividade da chuva (Valor R do USLE) e Fator Deficiência Bioclimática: Grau de umidade do solo e Risco de geadas.

\section{Modificações introduzidas no MICROLEIS/CERVATANA}

Nos parâmetros quantitativos: A estrutura do CERVATANA se caracteriza como matriz de pesos, ou seja, combinação linear ponderada de pesos conferidos a cada variável, segundo limites predeterminados.

Os critérios adotados para adaptação do aplicativo foram os seguintes:

a. Embora a estrutura do CERVATANA permita a utilização de $n+1$ variáveis, optou-se por manter o número original (Tabela 1), no caso, doze (12).

b. Substituição do parâmetro, quando este envolvesse dificuldade na obtenção ou quanto à relevância. Neste caso, as modificações foram as seguintes: 
Tabela 1. Alterações introduzidas no aplicativo original

\begin{tabular}{|c|c|c|c|}
\hline Variável/Parâmetro - CERVATANA & Peso & Variável/Parâmetro - SIAT & Peso \\
\hline Fator Relevo - Declividade & & Fator Relevo - Declividade & \\
\hline Nula ou Suave $(<7 \%)$ & 1 & Nula ou Suave $(<5 \%)$ & 1 \\
\hline Ligeira ou Moderada (7-15\%) & 2 & Ligeira ou Moderada (5-10\%) & 2 \\
\hline Forte $(15-30 \%)$ & 3 & Forte $(10-20 \%)$ & 3 \\
\hline Escarpada $(>30 \%)$ & 4 & Muito Forte $(>20 \%)$ & 4 \\
\hline Fator Solo - Prof. Útil & & Fator Solo - Prof. Útil & \\
\hline Elevada $(>75 \mathrm{~cm})$ & 1 & Elevada $(>200 \mathrm{~cm})$ & 1 \\
\hline Moderada $(50-75 \mathrm{~cm})$ & 2 & Moderada $(200-80 \mathrm{~cm})$ & 2 \\
\hline Escassa $(25-50 \mathrm{~cm})$ & 3 & $\operatorname{Rasa}(80-40 \mathrm{~cm})$ & 3 \\
\hline Superficial $(<25 \mathrm{~cm})$ & 4 & Superficial $(<40 \mathrm{~cm})$ & 4 \\
\hline Fator Solo - Tipo de Textura & & Fator Solo - Tipo de Textura & \\
\hline Equilibrada & 1 & Equilibrada & 1 \\
\hline \multirow[t]{2}{*}{ Ligeira ou Pesada } & 2 & Argilosa & 2 \\
\hline & & Arenosa & 3 \\
\hline . Fator Solo - Pedregosidade e/ou Afloramento & & Fator Solo - Pedregosidade e/ou Afloramento & \\
\hline Nula ou Ligeira $(<15 \%)$ & 1 & Nula ou Ligeira $(<10 \%)$ & 1 \\
\hline Ligeira a Moderada (15-40\%) & 2 & Ligeira a Moderada (10-20\%) & 2 \\
\hline Elevada $(>40 \%)$ & 3 & Elevada $(>20 \%)$ & 3 \\
\hline Fator Solo - Tipo de Drenagem & & Fator Solo - Tipo de Drenagem & \\
\hline Boa & 1 & Boa & 1 \\
\hline Moderada & 2 & Moderada & 2 \\
\hline Deficiente ou Excessiva & 3 & Deficiente ou Excessiva & 3 \\
\hline Fator Solo - Salinidade Total & & Fator Solo - Acidez/pH & \\
\hline Nula ou Ligeira $(<4$ mmhos $/ \mathrm{cm})$ & 1 & Nula ou Ligeira (7.0-6.0) & 1 \\
\hline Moderada (4-8 mmhos/cm) & 2 & Moderada $(6.0-5.0)$ & 2 \\
\hline Elevada $(8-12 \mathrm{mmhos} / \mathrm{cm})$ & 3 & Elevada $(5.0-4.0)$ & 3 \\
\hline Muito Elevada (> $12 \mathrm{mmhos} / \mathrm{cm})$ & 4 & Muito Elevada $(<4.0)$ & 4 \\
\hline Fator Risco à Erosão - Erodibilidade & & Fator Risco à Erosão - Erodibilidade & \\
\hline Ligeira & 1 & Ligeira & 1 \\
\hline Moderada & 2 & Moderada & 2 \\
\hline Elevada & 3 & Elevada & 3 \\
\hline . Fator Risco à Erosão - Declividade & & Fator Risco à Erosão - Declividade & \\
\hline$<15 \%$ & 1 & $<10 \%$ & 1 \\
\hline $15-30 \%$ & 2 & $10-20 \%$ & 2 \\
\hline$>30 \%$ & 3 & $>20 \%$ & 3 \\
\hline Fator Risco à Erosão - Densidade Vegetação & & Fator Risco à Erosão - Densidade Vegetação & \\
\hline Elevada & 1 & Elevada $(>80 \%)$ & 1 \\
\hline Moderada & 2 & Moderada $(80-40 \%)$ & 2 \\
\hline Nula & 3 & Baixa $(<40 \%)$ & 3 \\
\hline . Fator Risco à Erosão - Erosividade da Chuva & & Fator Risco à Erosão - Erosividade da Chuva & \\
\hline Ligeira $(<150)$ & 1 & Ligeira $(<700)$ & 1 \\
\hline Moderada $(150-200)$ & 2 & Moderada $(700-725)$ & 2 \\
\hline Forte $(200-300)$ & 3 & Forte $(725-750)$ & 3 \\
\hline Muito Forte $(>300)$ & 4 & Muito Forte $(>750)$ & 4 \\
\hline . Fator Deficiência Bioclimática & & Fator Deficiência Bioclimática & \\
\hline Grau de Umidade $(\mathrm{Ih}=\mathrm{P} / \mathrm{ETP})$ & & Grau de Umidade $(\mathrm{AD}=\mathrm{P}-\mathrm{ETP})$ & \\
\hline Suficiente $(=>1)$ & 1 & Excesso $(>1500)$ & 1 \\
\hline Moderado (1-1/2) & 2 & Suficiente (1500-350) & 2 \\
\hline Escasso $(1 / 2-1 / 3)$ & 3 & Moderado (350-200) & 3 \\
\hline Muito Escasso $(=<1 / 3)$ & 4 & Escasso $(<200)$ & 4 \\
\hline . Fator Deficiência Bioclimática & & Fator Deficiência Bioclimática & \\
\hline Risco de Geadas & & Risco de Geadas & \\
\hline Nulo ou Ligeiro $(=<2)$ & 1 & Nulo ou Ligeiro $(<2)$ & 1 \\
\hline Ligeiro a Moderado (2-5) & 2 & Ligeiro a Moderado (2-4) & 2 \\
\hline Elevado $(=>5)$ & 3 & Elevado $(>4)$ & 3 \\
\hline
\end{tabular}

b.1. Substituição do cálculo Grau de umidade de $\mathrm{Ih}=\mathrm{P} / \mathrm{ETP}$ por $\mathrm{AD}=\mathrm{P}$ - ETP, donde:
AD - Água Disponível
P - Precipitação
ETP - Evapotranspiração

b.2. Substituição da Salinidade total por Acidez (pH). Sendo um trabalho basicamente metodológico, desenvolvido no Estado de São Paulo, a acidez é muito mais relevante que salinidade, razão da substituição. Nas regiões semi-áridas, sem dúvida a salinidade poderia ser mantida. Não haveria 
impedimento, no entanto, de se acrescentar novas variáveis, ou seja, manter salinidade e acrescentar acidez.

c. Calibração dos intervalos de cada parâmetro empiricamente, tentativamente se aproximando do paradigma estabelecido, no caso, o trabalho "Aptidão Agrícola das Terras do Estado de São Paulo" de Oliveira \& Berg (1985). Quando considerado significante, aumentou-se o número de classes de um parâmetro. Em apenas um caso (Textura) isto aconteceu.

As modificações introduzidas aparecem na Tabela 1, ao lado dos parâmetros originais do sistema espanhol.

Na estrutura do programa: As modificações, neste caso, objetivaram: modernizar o sistema; aumentar a capacidade de processamento; tornar o sistema mais amigável e melhorar a apresentação dos resultados. A Tabela 2 apresenta as alterações.

A estrutura de cálculos do SIAT é a mesma do CERVATANA, ou seja, a entrada dos dados (12 variáveis) é feita quadrícula por quadrícula, que imediatamente é classificada em uma das quatro classes possíveis: $\mathrm{S}_{1}, \mathrm{~S}_{2}, \mathrm{~S}_{3}$ e N. A interface com o SIG permite que cada variável seja associada a um Plano de Informação (P.I.) de toda a área, no formato raster, num total de 12 P.I's. Codificados os atributos de cada quadrícula, em cada P.I. o sistema executa os cruzamentos necessários, resultando na ordenação das quatro classes possíveis para cada quadrícula. A articulação dos dados e planos de informações está representada na Figura 1.

\section{Interface com o IDRISI}

A interface engloba três etapas: do IDRISI para o SIAT; execução do SIAT; do SIAT para o IDRISI e espacialização dos resultados, e seu objetivo é fornecer uma ligação entre o SIAT e o SIG, permitindo que mapas digitalizados neste sistema possam servir de entrada de dados para o SIAT e que os resultados deste possam ser exportados para o SIG e tratados com todas as funções que este software possui, por exemplo: edição de mapa, display em cores de mapa na tela, impressão de mapa, descrição completa do tema, cruzamentos etc.

\section{Análise dos dados}

A comparação entre o mapa SIAT e o mapa de referência (Oliveira \& Berg, 1985) resulta em uma matriz de erro ou de contingência (Story \& Congalton, 1986). Os erros que podem aparecer neste caso podem ser de omissão e de inclusão sendo que, no primeiro caso, referem-se a amostras de certa classe dos dados SIAT, que não foram classificados como tal e, no segundo caso, a amostras de uma classe dos dados classificados e erroneamente classificados.

A amostragem delineada consiste de um número determinado de unidades de amostragem, que podem ser pontos, linhas ou áreas. Neste caso, foi utilizada uma grade, onde cada célula possui as dimensões de 2,0 x 2,0 mm que, para a escala 1:100.000, corresponde a uma área no terreno, de 4 ha $(200 \times 200 \mathrm{~m})$.

Tabela 2. Alterações na estrutura do praograma

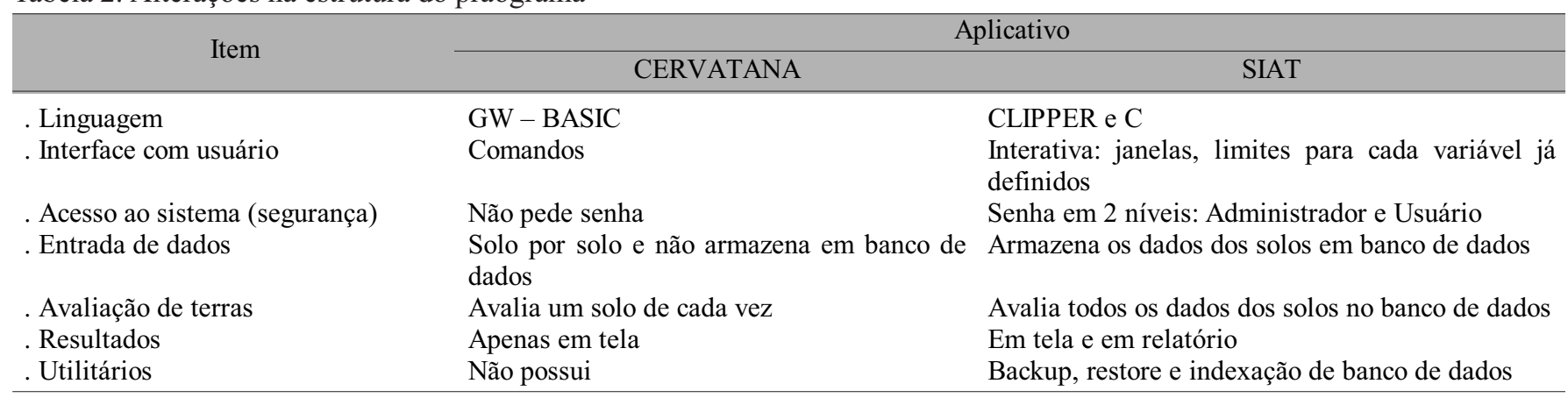

\section{Planos de Informações}

\begin{tabular}{|l|}
\hline BASE DE DADOS \\
\hline - Base Cartográfica \\
- Limites/divisas \\
- Hidrografia/drenagem \\
- Sistema viário \\
- Cidades e vilas \\
- Curvas de nivel \\
- Pedologia \\
- Mapa de solos \\
- Informações qualitativas \\
- Informações quantitativas \\
- Cobertura do Terreno \\
- Vegetação natural \\
- Agricultura \\
- Uso urbano \\
- Outros \\
- Clima \\
- Precipitação - mensal/anual \\
- Temperatura/geada \\
\hline
\end{tabular}

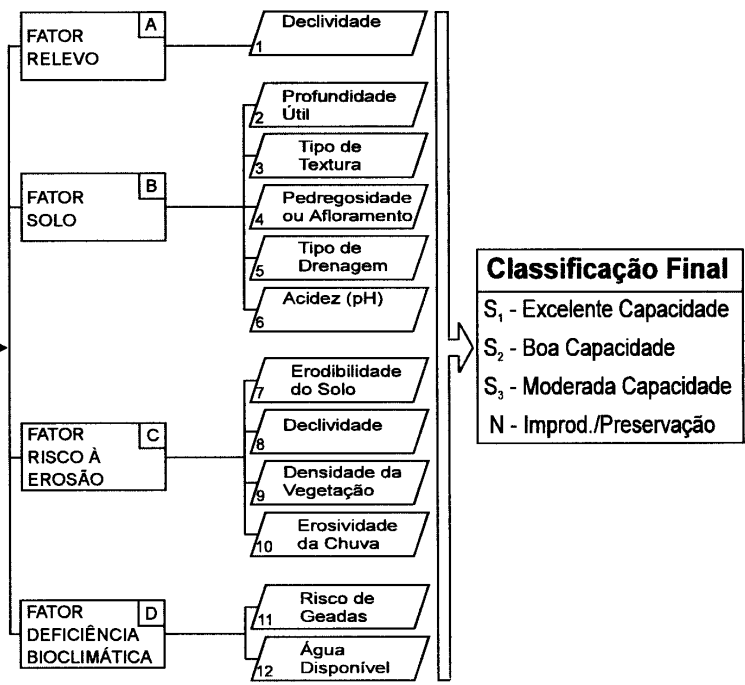

Figura 1. Articulação dos dados pelo SIAT 
Uma vez estabelecida a matriz, algumas medidas podem ser realizadas. De acordo com Veregin (1989) a proporção de células classificadas corretamente é calculada dividindo-se o número de amostras classificadas, posicionadas na diagonal da matriz, pelo número total de células avaliadas. Na avaliação da eficiência de cada calibração do sistema, a estatística de precisão/erro foi feita através de uma matriz de contingência. Para um limite de confiança, não menor que $85 \%$, utilizou-se da seguinte relação:

$$
\mathrm{p}=\tilde{\mathrm{p}}-1,645 \cdot \sqrt{\frac{\tilde{\mathrm{p}}-\tilde{\mathrm{q}}}{\mathrm{n}}}+\frac{50}{\mathrm{n}}
$$

onde:

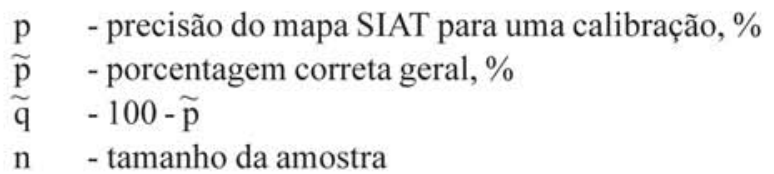

\section{RESULTADOS E DISCUSSÃO}

Conforme se observa na Figura 1, foram produzidos 12 mapas na forma raster, correspondentes às 12 variáveis do sistema SIAT, da forma como foram descritas anteriormente. Esses 12 planos de informações convenientemente processados no sistema, deram origem a uma classificação das terras, como mostra a Figura 2. O sistema permite ainda, para uma mesma área, a espacialização dos fatores limitantes, o que se configura como valiosa ferramenta para o planejamento de uso da terra.

Como já comentado, a calibração do modelo levou em conta principalmente o ajuste dos intervalos de cada parâmetro, tentativa, comparativamente ao trabalho de Oliveira \& Berg (1985) sobre "Aptidão agrícola das terras do Estado de São Paulo", escala 1:100.000.

Das várias ajustagens realizadas, aquela apresentada na Tabela 1 foi a que apresentou melhores resultados, a partir da aplicação da metodologia estatística já descrita. Neste caso, o erro médio de omissão foi de $4,4 \%$ e o erro médio de inclusão, de $3,8 \%$, para um resultado final de $97,2 \%$ de semelhança entre o mapa SIAT e o mapa de referência. Esta elevada semelhança é devida, provavelmente, ao fato do SIAT trabalhar apenas com 4 classes, induzindo a uma generalização significativa, evidenciando que o sistema é mais apropriado para escalas entre 1:50.000 e 1:100.000; no entanto, mesmo em escalas pequenas, o SIAT poderá proporcionar informações valiosas, considerando-se a precariedade dos mapeamentos a nível nacional. Pela flexibilidade que apresenta, é possível também ajustar-se o sistema para escala maiores, aumentando-se a precisão e se diminuindo a área. Para se chegar a este nível torna-se necessário restringir a amplitude dos parâmetros para certas variáveis, especialmente para aquelas associadas ao clima.

Um Manual do Usuário acompanha o aplicativo. Em breve, este manual estará disponibilizado na mesma mídia do SIAT.

\section{CONCLUSÕES}

Os resultados obtidos permitem as seguintes conclusões principais:

1. As modificações introduzidas no sistema original (CERVATANA) foram abrangentes que se pode afirmar que se tem, hoje, um novo sistema, chamado Sistema de Avaliação de Terras - SIAT.

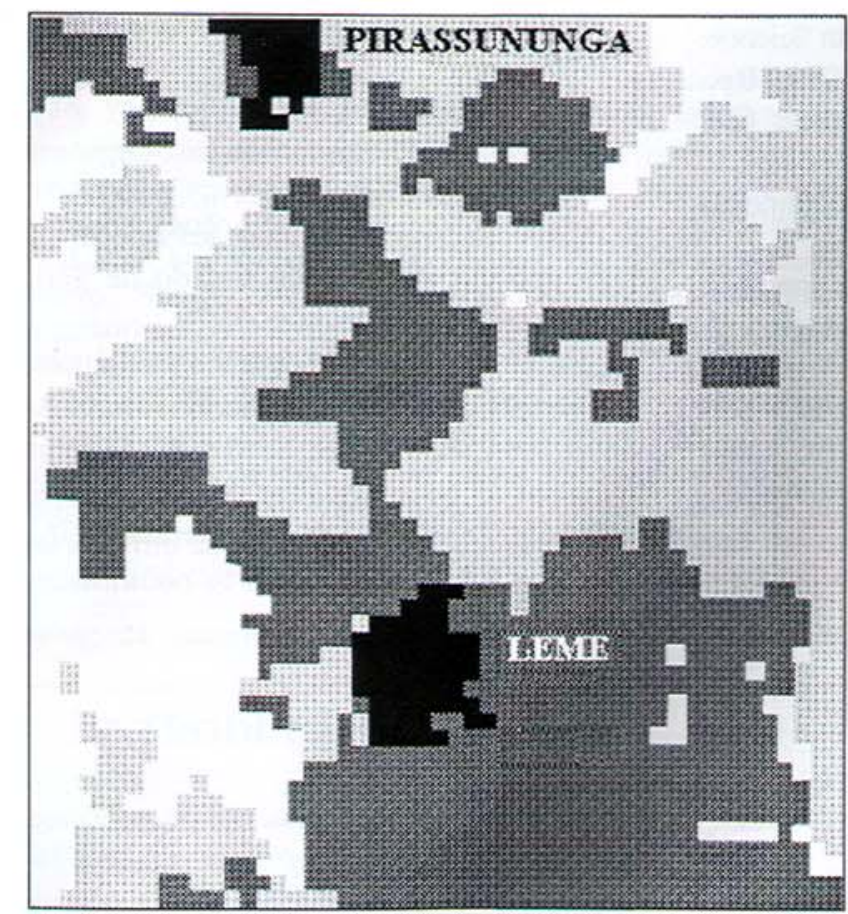

$47^{\circ} 30$

Figura 2. Classificação das terras, utilizando-se o aplicativo SIAT $22^{\circ} 00$

PLANO 13

CLASSIFICAÇÃO FINAL

CÓdIGo $\stackrel{\text { LEGENDA }}{\text { INTERPRETAÇÃo }}$

S1 EXCELENTE CAPACIDADE

S2 BOA CAPACIDADE

S3 MODERADA CAPACIDADE

ESCALA APROXIMADA

० $3.500 \mathrm{~m}$. 
2. Na versão atual, o SIAT é apropriado para escalas entre 1:100.000 e1:50.000.

3. Pela flexibilidade não só quanto ao número de variáveis mas, também, em relação ao ajuste na amplitude dos parâmetros, será possível melhorar-se a sensibilidade do SIAT, permitindo mapeamentos em escalas maiores.

4. Os resultados sugerem dar continuidade aos estudos, especialmente para outras regiões brasileiras.

\section{LITERATURA CITADA}

Anderson, L.T. Seven methods for calculating land capability/ suitability. Chicago: American Planning Association. Report $\mathrm{n}^{\circ}$ 402, 1987.20p.

Baudry, J. Interactions between agricultural and ecological systems at the landscape level. Agriculture, Ecosystems and Environment, Amsterdan, v.27, p.119-130, 1989.

Eastman, J.R. IDRISI user's guide. Worcester: Clark Univerisuty, 1997. 74p.

Elliot, E.T.; Cole, C.V. A perspective on agroecosystem science. Ecology. Ann Arbor. MI, v.70, n.11, p.1597-1602, 1989.

EMBRAPA - Empresa Brasileira de Pesquisa Agropecuária. Sistema brasileiro de classificação de solos. Brasília: Serviço de Produção da Informação,1999.412p.

Ferrari, G.A.; Magaldi, D. Land suitability evaluation for Mediterranean regions. Firenze: Instituto Agronômico per L'Oltremere, 1989. 60p.

Frank, A.V.; Engenhofer, M.J.; Kuhn, W. A perspective of GIS technology in the nineties. Photogrammetric Engineering and Remote Sensing, Falls Church, v.57, n.10, p.1431-1436, 1991.

IPT - Instituto de Pesquisa Tecnológica. Mapa geológico do Estado de São Paulo. Escala 1:50.000. 2 V. São Paulo, Instituto de Pesquisas Tecnológicas do Estado de São Paulo, 1981, $126 \mathrm{p}$.

ITC - International Institute for Aerial Survey and Earth Science. The LIS/GIS information system. Enshede: ITC, 1990. 10 p.

Keulen, H. Data and models in land resource management. ITC Journal, Enschede, v.12, n.3, p.363-368, 1990.

Koffler, N.F. Sistema de análise ambiental para planejamento agrícola. Versão 2.0. Rio Claro: CEAPLA, 1993.35p.

Lai, P. Feasability of geographic information systems approach for natural resource management. Environment Management, San Francisco, v.14, n.1, p.73-80, 1990.

Lanen, H.A.J.; Bregt, A.K., Hoobeek, R. Use of GIS, EC - Soil map to explore crop growth potentials. In: EC Land Evaluation Work Symposium, 1, 1988, Wageningen. Anais... Wageningen, 1988. p.80-90.
Lepsch, I.F. Manual para levantamento utilitário do meio físico e classificação de terras no sistema de capacidade de uso. Campinas: Sociedade Brasileira de Ciência do Solo, 1983. $175 \mathrm{p}$.

Lindhult, M.S.; Fabos, J.; Price, N. Using geographical information systems to assess conflicts between agriculture and development. Landscape and Urban Planning, San Francisco. v.16, n.4, p.333-343, 1988.

MacClean, A.L.; D’Avello, T.P.; Shetron, S.G. Digital soil maps in a GIS. Photogrammetric Engineering and Remote Sensing, Falls Church, v.59, n.3, p.223-228, 1993.

Oliveira, J.B.; Berg, M. Aptidão agrícola das terras do Estado de São Paulo. Quadrícula de Araras. Campinas: IAC, 1985. $60 \mathrm{p}$.

Oliveira, J.B.; Menk, J.R.F.; Barbieri, J.L., Rotta, C.L. Levantamento pedológico semidetalhado do Estado de São Paulo: Quadrícula de Araras. Campinas: IAC, 1982. 180p.

Ramalho, A.; Pereira, E.G.; Beek, K.J. Sistema de avaliação da aptidão agrícola das terras. Brasília: Embrapa, 1978, 70p.

Rosa, D.; Moreno, J.A.; Garcia, L.V.; Almorza, J. MicroLEIS: A microcomputer-based Mediterranean land evaluation information system. Soil Use and Management. Chicago. v.8, n.1, p.89-96, 1992.

Schrey, H.P. Criteria of GIS applications in soil Science. Environment and Urban Systems, San Francisco. v.16, n.2, p.203-207, 1992.

Secretaria da Agricultura. Áreas com possibilidade de expansão das culturas da cana-de-açúcar e da mandioca para a produção de álcool combustível no Estado de São Paulo. Campinas: IAC, 1976, 33p.

Secretaria da Indústria e Comércio. Rezoneamento das áreas para implantação de destilarias de álcool. São Paulo: SIC, 1980. 114p.

Setzer, J. Atlas climático ecológico do Estado de São Paulo. São Paulo. Comissão Internacional Bacia Paraná-Uruguai, 1966, 42p.

Story, M.; Congalton, R.G. Accuracy assessment: A user's perspective. Photogrammetric Engineering and Remote Sensing, Falls Church. v.52, n.3, p.397-399, 1986.

SUPLAN - Secretaria Nacional de Planejamento Agrícola. Mapa de aptidão agrícola do Estado de São Paulo. Brasília: Ministério da Agricultura, 1979.

Veregin, H. A taxonomy of error in spatial databases. Santa Barbara: National Center for Geographic Information Analysis, 1989. 113p.

Vieira, L.S.; Martins, J.S.; Santos, S.P. Sistema quantitativo para avaliar as possibilidades de utilização ecológica da terra. Brasília: MME/Projeto Radambrasil, 1976. 50p. 